\title{
Coherent mesoscopic transport through a quantum-dot embedded carbon nanotube ring threaded with magnetic flux
}

\author{
Hong-Kang Zhao ${ }^{1,2}$ and Jian Wang ${ }^{1}$ \\ 1. Department of Physics, The University of Hong Kong, Pokfulam Road, \\ Hong Kong, China \\ 2. Department of Physics, Beijing Institute of Technology, \\ Beijing 100081, China* \\ (October 30, 2003)
}

\begin{abstract}
We have investigated the coherent mesoscopic transport through a quantum dot (QD) embedded carbon nanotube ring (CNR) by employing the nonequilibrium Green's function (NGF) technique. The Landauer-Büttiker- like formula is presented to calculate the differential conductance and currentvoltage characteristics. Due to the interference of the electrons transporting in the two paths of CNR, the resultant conductivity of electron through the system is determined by the compound concrete structure of CNR-QD system. The tunneling current appears quantum behavior obviously in the small region of source-drain bias. The conductance is adjusted by the gate voltage $V_{g}$ and the magnetic flux $\phi$. The reversal resonance has been displayed versus the gate voltage, and it is symmetric about $V_{g}$ for the type I CNR, but it is asymmetric for the type II CNR. The phase inverse oscillations are also presented for the different types of CNRs.
\end{abstract}

73.63.Fg; 73.61.Wp; 73.22.-f;74.50.+r

The single-wall carbon nanotubes (SWCNs) provide prospective applications on the electronic nano-devices due to their electronic structures. One of the important property is the metal-semiconductor transition for different structures of SWCN [1,2], which has attracted much attention both experimentally and theoretically. This field opens up a new artificial laboratory to study onedimensional transport [3-8], such as the extensively investigated semiconductor quantum wire and hybrid device systems. The resonant tunneling behavior in the SWCN based magnetic tunneling junctions is an example of investigation, and the dynamic conductance in the SWCN system responded by an ac field exhibits significant difference from the dc conductance [9]. The evidence for the resonant transmissions has been observed experimently in SWCN system [3]. As the two ends of a SWCN connect to form a closed carbon nanotube ring (CNR), the detailed carbon nanotube structure takes the central role for the conducting behavior, since the CNR is quantized in both of the longitudinal and transversal directions [10-13]. The conductance of CNR is quite different even if for the type I and II armchair carbon nanotubes, and the metal-semiconductor transition is associated with the external magnetic flux [10,14-17]. As a pure carbon quantum dot is designed by introducing pentagon-heptagon defects in a carbon nanotube, the en- ergy difference, spatial confinement, and the number of discrete levels can be modified [18]. However, for the application point of view, it is more interesting to investigate the carbon nanotube based electronic device system. In this letter, we study the mesoscopic transport through the system with a quantum dot embedded in one arm of the CNR. The quantum dot can be taken as a nano-device applied with gate voltage, and the CNR is connected to two electrodes. A static magnetic flux is threaded through the CNR, which produces AharonovBohm-like effect. Due to the coherent interference and back scattering in the ring, the resulting conductance is quite different from the ring without the quantum dot. We show that the different types of CNR exhibit obviously diffferent behaviors with respect to the differential conductance and current-voltage characteristics.

We consider the situation that the leads broaden immediately at the connections to the CNR, and they are large enough to be considered as equilibrium electron reservoirs. A quantum dot with the energy $E_{d, j \sigma}^{(0)}$ is embedded in one arm of the CNR. The diameter ratio of the nanotube $d_{t}$ to the diameter $D_{t}$ of mesoscopic ring is much smaller than 1 , i.e., $\kappa=d_{t} / D_{t}<<1$. The CNR is described by the tight-binding Hamiltonian, and the two normal metal leads are described by the free electron grand canonical ensembles. In the diagonalized representation of CNR, the electronic properties can be determined by the total Hamiltonian of the system which is the summation of the sub-Hamiltonians and the tunneling interaction terms

$$
\begin{array}{r}
H=\sum_{\gamma k \sigma} \epsilon_{\gamma k} a_{\gamma, k \sigma}^{\dagger} a_{\gamma, k \sigma}+\sum_{j \ell \delta \sigma} \tilde{E}_{\delta, j \ell} c_{\delta \sigma, j \ell}^{\dagger} c_{\delta \sigma, j \ell} \\
+\sum_{j \sigma} E_{d, j \sigma} d_{j \sigma}^{\dagger} d_{j \sigma}+\sum_{i j p \delta \ell \sigma}\left(V_{\ell, i j}^{(p)} d_{\ell \sigma}^{\dagger} c_{\delta \sigma, i j}+H . c .\right) \\
+\sum_{\gamma k \sigma} \sum_{j \ell \delta}\left[R_{\gamma \delta, j \ell}^{*}(k) c_{\delta \sigma, j \ell}^{\dagger} a_{\gamma, k \sigma}+H . c .\right]
\end{array}
$$

where $a_{\gamma, k \sigma}^{\dagger}\left(a_{\gamma, k \sigma}\right), c_{\delta \sigma, j \ell}^{\dagger}\left(c_{\delta \sigma, j \ell}\right)$, and $d_{\ell \sigma}^{\dagger}\left(d_{\ell \sigma}\right)$ are the creation (annihilation) operators of electron in the two leads, the CNR, and quantum dot respectively, with $\gamma \in\{L, R\}$. In the energy $\tilde{E}_{\delta, j \ell}$ of the CNR, $\delta$ corresponds to the conduction and valence bands, respectively by choosing $\delta= \pm$. $R_{\gamma \delta, j \ell}(k)$ is interaction strength of 
electrons between the $\gamma$ th lead and CNR. $V_{\ell, i j}^{(p)}(\mathrm{p}=1,2)$ is the interaction strength between the quantum dot and ring. The interaction is associated with magnetic flux $\phi$ by $V_{\ell, i j}^{(p)}=V_{\ell, i j} \exp \left[2 \pi i(p-1) \phi / \phi_{0}\right]$, where $\phi_{0}=h / e$ is the flux quantum. We take the chemical potential of the right lead $\mu_{R}$ as the reference of energy measurement to ensure $\mu_{L}-\mu_{R}=e V$, where $V$ is the voltage between the two leads. $E_{d, j \sigma}=E_{d, j \sigma}^{(0)}-e V_{g}$ represents the energy levels of quantum dot modified by the gate voltage $V_{g}$ appling to the quantum dot, where $E_{d, j \sigma}^{(0)}$ is the $j$ th energy level of isolated quantum dot. In the Hamiltonian, $\sigma$ represents the spin index, however, for this spin-degenerate system the magnetic field is shielded in order not to affect the electron in the ring and quantum dot. The energy of CNR is intimately associated with the structure of concrete CNR. The CNR is formed by rolling a finite graphite sheet from the origin to the vectors $\mathbf{R}_{\mathrm{x}}=\mathrm{m}_{1} \mathbf{a}_{1}+\mathrm{m}_{2} \mathbf{a}_{2}$, and $\mathbf{R}_{\mathrm{y}}=\mathrm{p}_{1} \mathbf{a}_{1}+\mathrm{p}_{2} \mathbf{a}_{2}$ simultaneously. It is denoted by $\left(m_{1}, m_{2} ; p_{1}, p_{2}\right)$ as convention [10], and it satisfies the periodical boundary conditions along both of the longitudinal and transverse directions. The armchair $(m, m ;-p, p)$ CNR possesses the symmetry with armchair structure along the transverse direction and zigzag structure along the longitudinal direction. The two diameters for the armchair CNR are $d_{t}=3 b m / \pi, D_{t}=3^{1 / 2} b p / \pi$, where $b=1.44 \AA$. In the energy $\tilde{E}_{\delta, j \ell}$ of CNR, the indexes $j, \ell$ indicate the quantized energy levels as $j=1,2, \ldots, m$ and $\ell=1,2, \ldots, 2 p$. The armchair CNR is a metal as $p=3 \nu$ (type I CNR) with the zero energy gap $E_{g}=0$, while it is a semiconductor with narrow energy gap as $p=3 \nu \pm 1$ (type II CNR) where $\nu$ is an integer. For the type II CNR, the energy gap is determined by $E_{g}=2 \gamma_{0}|1-z(\xi)|$, where $z(\xi)=\cos (\xi)-3^{1 / 2} \sin (\xi), \xi=3^{1 / 2} b / D_{t}$, and $\gamma_{0}=3.033$ $\mathrm{eV}$.

The current tunneling from one lead to the other is determined by the Landauer-Büttiker-like formula [19], which can be derived from nonequilibrium Green's function (NGF) technique[20]

$$
I=\frac{e}{h} \sum_{\sigma} \int d \epsilon T_{\sigma}(\epsilon)\left[f_{L}(\epsilon)-f_{R}(\epsilon)\right]
$$

The transmission coefficient $T_{\sigma}(\epsilon)$ contains the scatterings from the quantum dot and CNR. We are interested in the symmetric system in the wide-band limit where the line-width $\Gamma_{L}=\Gamma_{R}=\Gamma$. The transmission coefficient is given by $T_{\sigma}(\epsilon)=-\Gamma \sum_{m n \delta} \operatorname{Im} G_{\delta \sigma, m n}^{r}(\epsilon)$, where $G_{\delta \sigma, m n}^{r}(\epsilon)$ is the Green's function of the CNR defined as $G_{\delta \sigma, m n}^{r}\left(t, t^{\prime}\right)=-\frac{i}{\hbar} \theta\left(t-t^{\prime}\right)\left\langle\left[c_{\delta \sigma, m n}(t), c_{\delta \sigma, m n}^{\dagger}\left(t^{\prime}\right)\right]_{+}\right\rangle$. The Fourier transformed Green's function of the CNR is given by $G_{\delta \sigma, m n}^{r}(\epsilon)=g_{\delta, m n}^{r}(\epsilon)\left[1+4 \cos ^{2}\left(\pi \phi / \phi_{0}\right) \sum_{\ell}\left|V_{\ell, m n}\right|^{2}\right.$ $\left.g_{\delta, m n}^{r}(\epsilon) G_{d \ell, \sigma}^{r}(\epsilon)\right]$, where $G_{d \ell, \sigma}^{r}$ is the Green's function of the quantum dot. The derivation by NGF results in

$$
\begin{aligned}
T_{\sigma}(\epsilon)=\sum_{m n \delta} T_{m n \delta}^{(0)}(\epsilon)[1 & +\frac{4}{\Gamma} T_{m n \delta}^{(0)}(\epsilon) \sum_{\ell}\left|V_{\ell, m n}\right|^{2} \\
& \left.\times K_{\ell, m n \sigma}^{(\delta)}(\epsilon) \cos ^{2}\left(\pi \phi / \phi_{0}\right)\right]
\end{aligned}
$$

where $K_{\ell, m n \sigma}^{(\delta)}(\epsilon)=\left\{1-\left[\left(\epsilon-E_{\delta, m n}\right) / \Gamma\right]^{2}\right\} \operatorname{Im} G_{d \ell, \sigma}^{r}(\epsilon)+$ $2\left[\left(\epsilon-E_{\delta, m n}\right) / \Gamma\right] \operatorname{Re} G_{d \ell, \sigma}^{r}(\epsilon)$. The Green's function of the quantum dot is $G_{d \ell, \sigma}^{r}(\epsilon)=1 /\left[\epsilon-E_{d, \ell \sigma}-\Pi^{r}(\epsilon, \phi)\right]$, where $\Pi^{r}(\epsilon, \phi)=4 \sum_{\delta m n}\left|V_{\ell, m n}\right|^{2} \cos ^{2}\left(\pi \phi / \phi_{0}\right) g_{\delta, m n}^{r}(\epsilon)$. The Green's function of the ring without the embedded quantum dot is $g_{\delta, m n}^{r}(\epsilon)=1 /\left(\epsilon-E_{\delta, m n}+i \Gamma\right)$, and $T_{m n \delta}^{(0)}(\epsilon)$ is the transmission coefficient of the system without the embedded quantum dot $T_{m n \delta}^{(0)}(\epsilon)=\Gamma^{2}\left|g_{\delta, m n}^{r}(\epsilon)\right|^{2}$. The quantum dot contributes a quantity to the total transmission coefficient due to the scattering procedure of electron in the ring with the quantum dot. The first part of the transmission coefficient $T_{m n \delta}^{(0)}(\epsilon)$ describes the situation that the electrons tunneling through the mesoscopic ring without scattering with the quantum dot. As a result, the total transmission of electron through the mesoscopic system is determined by the interference of electron wave functions in the ring, which include the incident electron wave functions and the scattering wave functions of electron in the ring. The transmission is related to the magnetic flux $\phi$. The scattering of electron caused by the quantum dot takes its maximum value as $\phi=n \phi_{0}$, and this contribution becomes zero as $\phi=(1 / 2+n) \phi_{0}$, $(n=0, \pm 1, \pm 2, \ldots)$. The latter situation corresponds to the effect that the electrons transport through the CNR without the quantum dot completely. The resonant tunneling takes place in the Breit-Wigner form through each channel of the CNR. The resonance channels for this case are located at the energy levels of CNR $\epsilon=E_{\delta, m n}$. However, as $\phi \neq(1 / 2+n) \phi_{0}$, the transmission of electron is very complicated, the electrons transporting through the two arms of CNR contribute to the conductance consequently. The quantum dot opens the tunneling channels at $\epsilon=E_{d, \ell \sigma}+\operatorname{Re} \Pi^{r}(\epsilon, \phi)$, and the CNR also provides tunneling channels for electron to transport at $\epsilon=E_{\delta, m n}$. What path the electron is favorite to choose depends on the energy of electron in the leads. The contribution of quantum dot to the current is also associated with the interaction strengths $V_{\ell, m n}$. As $V_{\ell, m n}$ is strong enough, the quantum dot plays an important role to the mesoscopic transport. As $V_{\ell, m n}=0$ the quantum dot is disconnected to the CNR, and there is no contribution of the quantum dot to the transport. As the levels of quantum dot and CNR match with each other, the CNR and quantum dot open the resonant tunneling channels, and interference of electron coming from the two arms determines the resultant conductance. The transmission coefficient $T_{\sigma}(\epsilon)$ is an even and periodic function of magnetic flux, i.e., $T_{\sigma}(\phi)=T_{\sigma}(-\phi)$, and $T_{\sigma}(\phi)=T_{\sigma}\left(\phi+n \phi_{0}\right)$.

For the numerical calculations, we choose the parameters as $\Gamma=3.033 \mathrm{meV}$, and $V_{\ell, m n}=3.033 \mathrm{meV}$ for the armchair (m,m;-p,p) CNR-QD system at zero tempera- 
ture. The quantum dot is assumed to possess five symmetric levels $E_{d, \ell \sigma}^{(0)}=0, \pm 0.0025 \gamma_{0}, \pm 0.005 \gamma_{0}$. We take $G_{0}=2 e^{2} / h$ as the measurement scale of conductance, and $I_{0}=2 e \gamma_{0} / h=2.35 \times 10^{-4} \mathrm{~A}$ as the scale of tunneling current. We consider the type I $(5,5 ;-75,75)$ and type II $(5,5 ;-74,74)$ CNRs as examples, where the diameter ratio $d_{t} / D_{t} \approx 0.117$, and the energy gap of type II isolated CNR $E_{g} \approx 0.147 \mathrm{eV}$. However, the calculations contain main information for the two types of CNR as $d_{t} / D_{t}<<1$. Figure 1 displays the differential conductance versus the source-drain bias at zero temperature. The CNR possesses tunneling channels in a large region of energy, but the quantum dot only opens several discrete resonant levels for electron to tunnel. For the embedded quantum dot, $\operatorname{Re} \Pi^{r}(\epsilon, \phi)$ acts as novel channels in addition to $E_{d, \ell_{\sigma}}^{(0)}$. Therefore, the tunneling channels in the quantum dot extend to a large region of energy to overlap some tunneling channels of the isolated CNR. This causes the degeneration of differential conductance in a large region of $\mathrm{eV}$.

We display the tunneling current and differential conductance versus the source-drain bias $e V$ in Fig. 2. The current exhibits quantum steps obviously associated with the structure of CNR. For the type I CNR, the conductance shows a resonant peak at $V=0$, which indicates that there exists tunneling current as $V \neq 0$. This system is metallic in which the energy gap $E_{g}=0$, and it signifies that the tunneling levels of QD and CNR match each other at $e V=0$. For the type II CNR, the conductance resonates at $\mathrm{eV}= \pm 0.025 \gamma_{0}, \pm 0.05 \gamma_{0}$. This system is a semiconductor with the energy gap $E_{g} \approx 0.147 \mathrm{eV}$.

The differential conductance $d I / d V$ oscillating with respect to the magnetic flux $\phi$ is presented in Fig. 3. The conductance is a periodic and even function of $\phi$ with period $\phi_{0}$. The magnitude of conductance is adjusted by the gate voltage, and it shifts as the gate voltage increases shown in (a). The magnitude and shape of oscillation are strongly dependent on CNR. The zero-biased differential conductance of type I CNR is large, and it oscillates smoothly. This reflects the metallic AharonovBohm-like behavior. The differential conductance for the type II CNR is quite different from that of type I CNR. As $e V=0, d I / d V$ is very small, and there exists abrupt changes at $\phi=\phi_{0}(1 / 2+n)(n=0, \pm 1, \pm 2, \ldots)$. For this case the off-resonant tunneling through the quantum dot dominates the mesoscopic transpot. As the source-drain bias is nonzero, the conductance recovers the smooth oscillating behavior shown in diagram (b), but with the inverse phase oscillating structure compared with the zerobiased conductance of type I system.

The differential conductance $d I / d V$ versus the gate voltage $V_{g}$ is depicted in Fig. 4 as $\phi=0$. Diagrams (a) and (b) show the situations for the tpye I and type II CNRs with different source-drain biases $\mathrm{eV}=0$ and $e V=0.025 \gamma_{0}$, respectively. The magnitude and behav- ior of $\mathrm{dI} / \mathrm{dV}$ versus $V_{g}$ are dependent on the structure of CNR sensitively, and the inverse resonances are observed. For the type I CNR system, the inverse resonance is symmetric about $e V_{g}=0$, while for the type II CNR system the inverse resonance is asymmetric. The inverse resonance signifies that the quantum dot contributes a negative resonant conductance to the total one.

In summary, the differential conductance and I-V characteristics have been presented for the type I and II CNR systems. The transport properties are strongly associated with the structure of CNR, and the tunneling current appears quantum behavior obviously in the small region of source-drain bias. The conductance is a periodic function of $\phi$, and it is adjusted by the gate voltage. The reverse resonance has been displayed versus the gate voltage. The reverse resonance is symmetric about $e V_{g}$ for the type I CNR, but it is asymmetric for the type II CNR. The phase inverse oscillations are also observed for different types of CNR, and the quantum dot plays an important role in the mesoscopic transport. This system can be taken as an interference CNRFET controlled by the external magnetic flux and gate voltage.

This work was supported by a RGC grant from the SAR Government of Hong Kong under Grant No. HKU 7091/01P, and by the National Natural Science Foundation of China under the Grant No. 10375007.

[1] S. Iijima, Nature (London) 354, 56 (1991).

[2] J.W. Mintmire, B.I. Dunlap, and C.T. White, Phys. Rev. Lett. 68,631 (1992).

[3] S.J. Tans, M.H. Devoret, H. Dai, A. Thess, R.E. Smalley, L.J. Geerligs, and C. Dekker, Nature (London) 386, 474 (1997).

[4] C. Zhou, J. Kong, and H. Dai, Phys. Rev. Lett. $\mathbf{8 4 , 5 6 0 4}(2000)$.

[5] T. W. Odom, J.L. Huang, P. Kim, and C.M. Lieber, Nature (London) 391, 62 (1998).

[6] S.J. Tans, A.R.M. Verschueren, and C. Dekker, Nature (London) 393, 49 (1998).

[7] A. Bachtold, C. Strunk, J.P. Salvetat, J.M. Bonard, L. Forró, T. Nussbaumer and C. Schönenberger, Nature (London) 397, 673 (1999).

[8] Y. Xue, and S. Datta, Phys. Rev. Lett. 83, 4844 (1999).

[9] H. Mehrez, J. Taylor, H. Guo, J. Wang, and C. Roland, Phys. Rev. Lett. 84, 2682 (2000); C. Roland, M. B. Nardelli, J. Wang, and H. Guo, Phys. Rev. Lett. 84, 2921 (2000).

[10] M.F. Lin, and D.S. Chuu, Phys. Rev. B 57,6731 (1998).

[11] B.I. Dunlap, Phys. Rev. B 46, 1933 (1992). 
[12] S. Itoh, S. Ihara, and J. Kitakami, Phys. Rev. B 47, 1703 (1993); ibid. 47, 12908 (1993).

[13] R.C. Haddon, Nature (London)388,31 (1997).

[14] J.P. Lu, Phys. Rev. Lett. 74, 1123 (1995).

[15] S. Roche, G. Dresselhaus, and M.S. Dresselhaus, and R. Saito, Phys. Rev. B 62, 16092 (2000).

[16] H.K. Zhao, Phys. Lett. A 308, 226 (2003); H.K. Zhao, Phys. Lett. A 310,207 (2003).

[17] H.K. Zhao, Eur. Phys. J. B 33,365 (2003).

[18] L. Chico, M.P. López, Sancho, and M.C. Muñoz, Phys. Rev. Lett. 81, 1278 (1998).

[19] R. Landauer IBM J. Res. Dev. 1, 223 (1957); M. Büttiker, Phys. Rev. Lett. 57, 1761 (1986).

[20] A.-P. Jauho, N.S. Wingreen and Y. Meir, Phys. Rev. B 50, 5528 (1994).

\section{Figure Captions}

Fig. 1, The differential conductance $d I / d V$ versus source-drain bias $e V$ as $V_{g}=0, \phi=0$. Diagrams (a), (b) are associated with the type I $(5,5 ;-75,75)$ and type II $(5,5 ;-74,74)$ CNRs, respectively.

Fig. 2, The I-V characteristics and differential conductance $d I / d V$ versus source-drain bias $e V$ as $V_{g}=0$ and $\phi=0$ corresponding to (a) and (b). The dotted and solid curves are associated with $(5,5 ;-75,75)$ and $(5,5 ;-74,74)$ CNRs, respectively.

Fig. 3, The differential conductance $d I / d V$ versus the magnetic flux $\phi$. Diagram (a) displays the conductance of $(5,5 ;-75,75) \mathrm{CNR}$ at $\mathrm{eV}=0$ with the solid, dotted and dashed curves corresponding to $e V_{g}=$ $0,0.001 \gamma_{0}, 0.002 \gamma_{0}$. Diagram (b) is the conductance of $(5,5 ;-74,74) \mathrm{CNR}$ as $e V=0.025 \gamma_{0} V_{g}=0$.

Fig. 4. The differential conductance $d I / d V$ versus gate voltage $V_{g}$ as $\phi=0$. Diagrams (a) and (b) correspond to the conductances of $(5,5 ;-75,75)$ CNR with $\mathrm{eV}=0$ and $(5,5 ;-74,74)$ CNR with $\mathrm{eV}=0.025 \gamma_{0}$, respectively. 